\title{
Theoretical Insights into the Hydrogen Evolution Activity of Layered Transition Metal Dichalcogenides
}

\author{
Charlie Tsai, ${ }^{a, b}$ Karen Chan, ${ }^{a, b}$ Jens K. Nørskov, ${ }^{a, b}$ Frank Abild-Pedersen ${ }^{* b}$ \\ ${ }^{a}$ Department of Chemical Engineering, Stanford University, Stanford, California 94305, USA \\ ${ }^{b}$ SUNCAT Center for Interface Science and Catalysis, SLAC National Accelerator Laboratory, 2575 Sand Hill Road, Menlo \\ Park, California 94025, USA
}

\begin{abstract}
A thermodynamic descriptor-based approach using density functional theory calculations was used to investigate the activity and stability of 26 different transition metal dichalcogenide catalysts for the hydrogen evolution reaction (HER). We considered variations in the transition metal ( $\mathrm{Ti}, \mathrm{V}, \mathrm{Nb}, \mathrm{Ta}, \mathrm{Mo}, \mathrm{W}, \mathrm{Pd}, \mathrm{Pt})$, the chalcogen ( $\mathrm{S}$ and $\mathrm{Se})$, the crystal structure $(1 \mathrm{~T}$ and $2 \mathrm{H})$, and the surface termination (basal plane or edge). We find that the HER activity is strongly related to the stability of the catalyst, setting practical limitations on their potential application in HER. For the basal planes, the metallicity is found to be the most important parameter in determining the activity rather than structure or composition. However, systematic improvements in activity are strongly limited by a decrease in stability. For the edges, the activity and stability relationship are similar regardless of structure or chalcogen, and it is possible to achieve optimal hydrogen binding with a stable surface. Nudged elastic band calculations were carried out to probe the possible mechanisms for HER; the insurmountably high barrier for the Tafel mechanism suggests that HER may occur solely via the Volmer-Heyrovsky route for these materials.
\end{abstract}

KEYWORDS: Transition metal dichalcogenide, hydrogen evolution reaction, density functional theory, basal plane, edges, electrochemistry

\section{INTRODUCTION}

$\mathrm{H}_{2}$ is increasingly being considered as an energy carrier for the intermittent solar energy,[1,2] which could be achieved by splitting water using electricity generated through sunlight or wind power. To this end, efficient catalysts are required for the hydrogen evolution reaction (HER), where protons from solution combine with electrons at the electrode to form $\mathrm{H}_{2}$ gas. The scarce and expensive Pt-group metals are still the most active catalysts for HER, so efficient, earth-abundant, and low-cost alternatives are needed. Following the success of nano-structured $2 \mathrm{H}-\mathrm{MoS}_{2}$, whose edge sites were first predicted by theory [3-5] and then proven in several experimental studies to be active for HER, [4,6-10] other members of the layered transition metal dichalcogenides (TMD) have been studied recently as a class of efficient non-precious alternatives to Pt. [6,7,1115] Recent advances in applying TMD catalysts to more complicated reactions such as $\mathrm{CO}_{2}$ electroreduction[16,17] have further established TMD's as a potentially versatile class of catalysts.

The TMD's have the chemical composition of $\mathrm{MX}_{2}$, where $\mathrm{M}$ is the metal and $\mathrm{X}$ is the chalcogen, and exist primarily in the $2 \mathrm{H}$ trigonal prismatic or the $1 \mathrm{~T}$ octahedral coordination (Figure 1). Strong chemical bonds hold the atoms together in-plane but the layers are held together through weak van der Waals interactions out-of-plane.[5,18] For metals in groups 4, 7, and 10 in the periodic table, the TMD's prefer the 1T phase in the bulk form, whereas for group 6 metals, the 2H phase is preferred. [19] Group 5 metals could take on either $1 \mathrm{~T}$ or $2 \mathrm{H}$ phase. They could be either metallic (groups 4 and 5) or semiconducting (groups 6,7, and 10). Since the metallic edges of TMD's were identified as the active sites for catalysis on single and few-layered $2 \mathrm{H}-\mathrm{MoS}_{2}, 2 \mathrm{H}-\mathrm{WS}_{2}, 2 \mathrm{H}-\mathrm{MoSe}_{2}$, and $2 \mathrm{H}-\mathrm{WSe}_{2}$, there has been a strong focus on nano-structuring these materials to maximally expose the edge sites and optimize activity. $[8,10-12,20]$ However, recent work on the metastable $1 \mathrm{~T}-\mathrm{MoS}_{2}$ and $1 \mathrm{~T}-\mathrm{WS}_{2}$ metallic structures have suggested that the basal planes are also active, leading to a dramatic increase in catalytic activity.[14,21-23] This raises the question of how the activity of TMD catalysts is generally determined. In order to further exploit these materials and design new and active catalysts, it is crucial to understand the specific role of the transition metal, the chalcogen, the metallicity, the structure, and the surface of the TMD in determining the overall catalytic activity and stability. A theoretical evaluation of the activity-stability relationships for these catalysts would establish the potential for further exploring these materials for HER.

In this study, we used a descriptor-based approach to evaluate the HER activity and stability of the basal planes and the edges of single layered TMD catalysts. For several $1 \mathrm{~T}-\mathrm{MX}_{2}$ and $2 \mathrm{H}-\mathrm{MX}_{2}$ surfaces $(\mathrm{M}=\mathrm{Ti}, \mathrm{V}, \mathrm{Nb}, \mathrm{Ta}, \mathrm{Mo}, \mathrm{W}, \mathrm{Pd}$, or Pt. $\mathrm{X}=$ $\mathrm{S}$ or Se) the free energy of hydrogen adsorption $\Delta G_{\mathrm{H}}$ (descriptor for activity) was determined as a function of HX adsorption $\Delta G_{\mathrm{HX}}$ (descriptor for stability). The observed inverse relationship between $\mathrm{H}$ binding and $\mathrm{HX}$ binding on the surfaces suggest 
that improvements in catalytic activity are always limited by the decrease in stability. For the basal planes of all TMD's, the metallicity of the surface determines the activity-stability relationship, with metallic basal planes having much stronger and more thermo-neutral $\Delta G_{\mathrm{H}}$ for similar stabilities.[9,24-26] For the edge sites, the activity and stability have a similar inverse correlation regardless of the composition, bulk metallicity, or specific edge termination (1T-edge, $2 \mathrm{H}$ M-edge, or $2 \mathrm{H} \mathrm{X}$ edge).

\section{THEORETICAL APPROACH}

The hydrogen adsorption free energy, $\Delta G_{\mathrm{H}}$, has been shown to be a successful descriptor of the HER activity, where a value of $\Delta G_{\mathrm{H}}$ close to thermo-neutral results in the most active catalyst. [9,24,25,27] An optimal catalyst would bind $\mathrm{H}$ neither too strongly nor too weakly. This was first established for pure transition metals, then successfully applied to transition metal alloys,[28] the edge sites of $2 \mathrm{H}-\mathrm{MoX}_{2}$ and $\mathrm{WX}_{2}$ catalysts $(\mathrm{X}=\mathrm{S}, \mathrm{Se}),[6,8,12,26]$, and transition metal carbides.[18] We apply this approach here for the study of activity on the TMD catalysts as well. Under reducing conditions, the vacancy formation reaction $\left(\mathrm{H}_{2} \mathrm{X}\right.$ desorption) will compete with hydrogen evolution since they both involve hydrogen combining with the $\mathrm{H}-\mathrm{X}$ group in the first step. In hydrogen evolution, the adsorbed $\mathrm{H}$ in the $\mathrm{H}-\mathrm{X}$ group will combine with another $\mathrm{H}$ atom to form $\mathrm{H}_{2}$, whereas in $\mathrm{H}_{2} \mathrm{X}$ desorption, another $\mathrm{H}$ atom combines with the $\mathrm{H}-\mathrm{X}$ group to be removed as $\mathrm{H}_{2} \mathrm{X}$. Hence, we have chosen the HX adsorption free energy as a descriptor for the stability of the catalyst under reaction conditions. If HX is weakly bound, desorption as $\mathrm{H}_{2} \mathrm{X}$ will be more likely and degradation of the catalyst could limit its practical implementation. An $\mathrm{H}_{2} \mathrm{X}$ pressure of $10^{-6}$ bar was chosen following standard corrosion resistance.[29,30] A more negative $\Delta G$ indicates a more exothermic reaction in all cases. The adsorption energies are defined as in previous studies,[4,6,31] and their definitions as well as computational details are included in the supporting information. We considered the (0001) basal plane as well as the M-edge and $\mathrm{X}$-edge of the $2 \mathrm{H}$ structures, and the edge of the 1T structures (both edges on the 1T are the same).

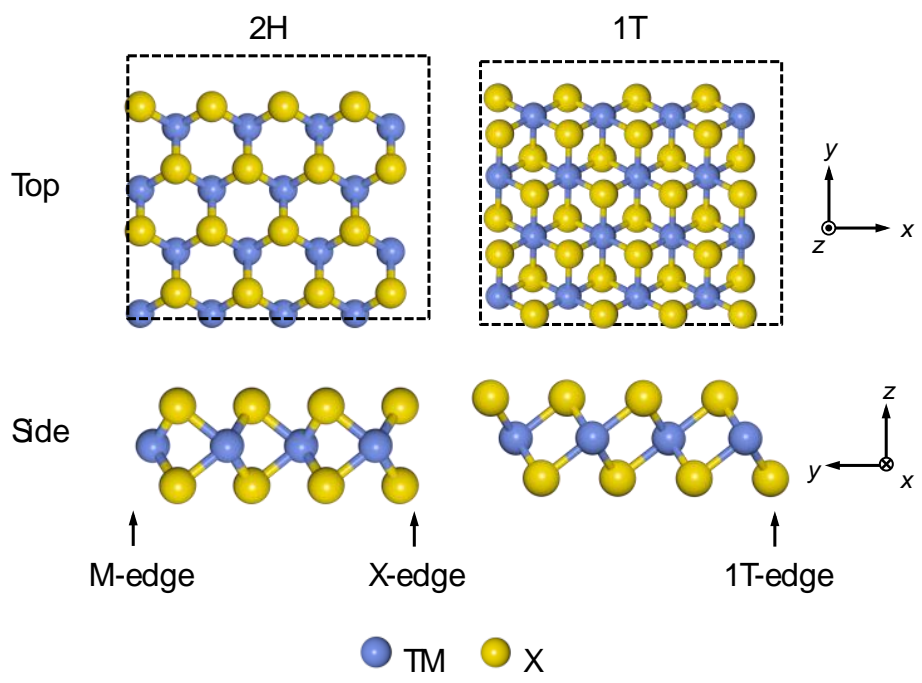

Figure 1. Calculation unit cells for both the $2 \mathrm{H}$ and $1 \mathrm{~T}$ structures of TMD basal planes, showing the top and side views, where $\mathrm{TM}=\mathrm{Ti}, \mathrm{V}, \mathrm{Nb}, \mathrm{Ta}, \mathrm{Mo}, \mathrm{W}, \mathrm{Pd}$, and Pt; X $=\mathrm{S}$ or Se. The three types of edges, the $1 \mathrm{~T}$-edge, the $2 \mathrm{H} \mathrm{M}$-edge, and the $2 \mathrm{H} \mathrm{X}$-edge are indicated by the arrows.

It is more complicated to model the edge sites, as their specific structure (the coverage of chalcogen atoms on the edge) is highly dependent upon the operating conditions[1] and there are two types of edge terminations for the $2 \mathrm{H}$-structure, the $\mathrm{M}$ edge and the X-edge. The concentration of S or Se atoms on the edge will vary among the structures under the same operating conditions. To model the HER activity of the edge sites under realistic operating conditions, we explicitly calculated a wide range of chalcogen and hydrogen coverages on the edge sites for the $2 \mathrm{H} \mathrm{M}$-edge, the $2 \mathrm{H} \mathrm{X}$-edge, and the $1 \mathrm{~T}$ edge. In reality, desorption of S or Se is kinetically limited by hydrogen evolution since both reactions involve hydrogen adsorbed on sulfur in the first step. However, since adsorption energies are known to scale with kinetic barriers, we use a thermodynamic analysis as a first approximation. Our approach is to start with the most thermodynamically favorable edge structure at 0 $\mathrm{V}_{\mathrm{RHE}}$ and assume that the steady-state structure is where $\mathrm{H}_{2}$ evolution is more exergonic than either $\mathrm{H}_{2} \mathrm{~S}$ desorption or further $\mathrm{H}$ adsorption. Additional information on the methods have been described in detail in several recent studies.[6,16,32,33] The steady-state structures are summarized in the supplementary information.

\subsection{Calculation Details}

All structures were calculated using plane-wave density functional theory (DFT) employing periodic boundary conditions. The Bayesian error estimation exchange-correlation functional with long-range interactions (BEEF-vdW) was used in order to properly account for the van der Waals interactions. Like the RPBE functional, the BEEF-vdW functional is optimized for chemisorption energies. A plane-wave cutoff of $500 \mathrm{eV}$ and a density cutoff of $5000 \mathrm{eV}$ were used, whereas the Brillouin zone was sampled using a Monkhorst-Pack $4 \times 4 \times 1 k$-point grid for the basal plane calculations, and a $2 \times 1 \times 1 k$-point grid for 
the edges. Both plane wave cutoffs and $k$-point grid density were checked for convergence with respect to chemisorption energies. The energy of the transition state for the Tafel mechanism was determined using climbing image nudged elastic band (NEB) calculations.[34]

\section{RESULTS AND DISCUSSION}

The hydrogen evolution activity of layered TMD could be dependent on the surface site: either the basal plane or the edge sites. Indeed, scanning tunneling microscopy studies have been used to definitively correlate the activity of $2 \mathrm{H}-\mathrm{MoS}_{2}$ with the number of edge sites [8]. Surface oxidation experiments have also been performed to indicate that basal plane of 1T$\mathrm{MoS}_{2}$ is active[21]. Herein, we explore the role of the metal, the chalcogen, the structure, and the surface termination in determining the activity and stability of TMD catalysts.

\subsection{Basal Plane}

The p-projected densities of states (p-DOS) were calculated on the chalcogen atom on the basal plane of the TMD's to determine their metallicity (Figure 2). All group 5 TMD's were found to be metallic regardless of their structure (2H or 1T), with states at the Fermi level. Group 4 TMD's were also found to be metallic, but with fewer states near the Fermi level compared to group 5. For group 6 TMD's, the $2 \mathrm{H}$ basal planes were found to be semiconducting with a band-gap, whereas the 1T basal planes were found to be metallic. Group 10 basal planes are also semiconducting in the 1T structure with a band-gap. All these results on the single layered basal planes confirm what is known about their bulk metallicity from previous experimental studies. [3,5] 


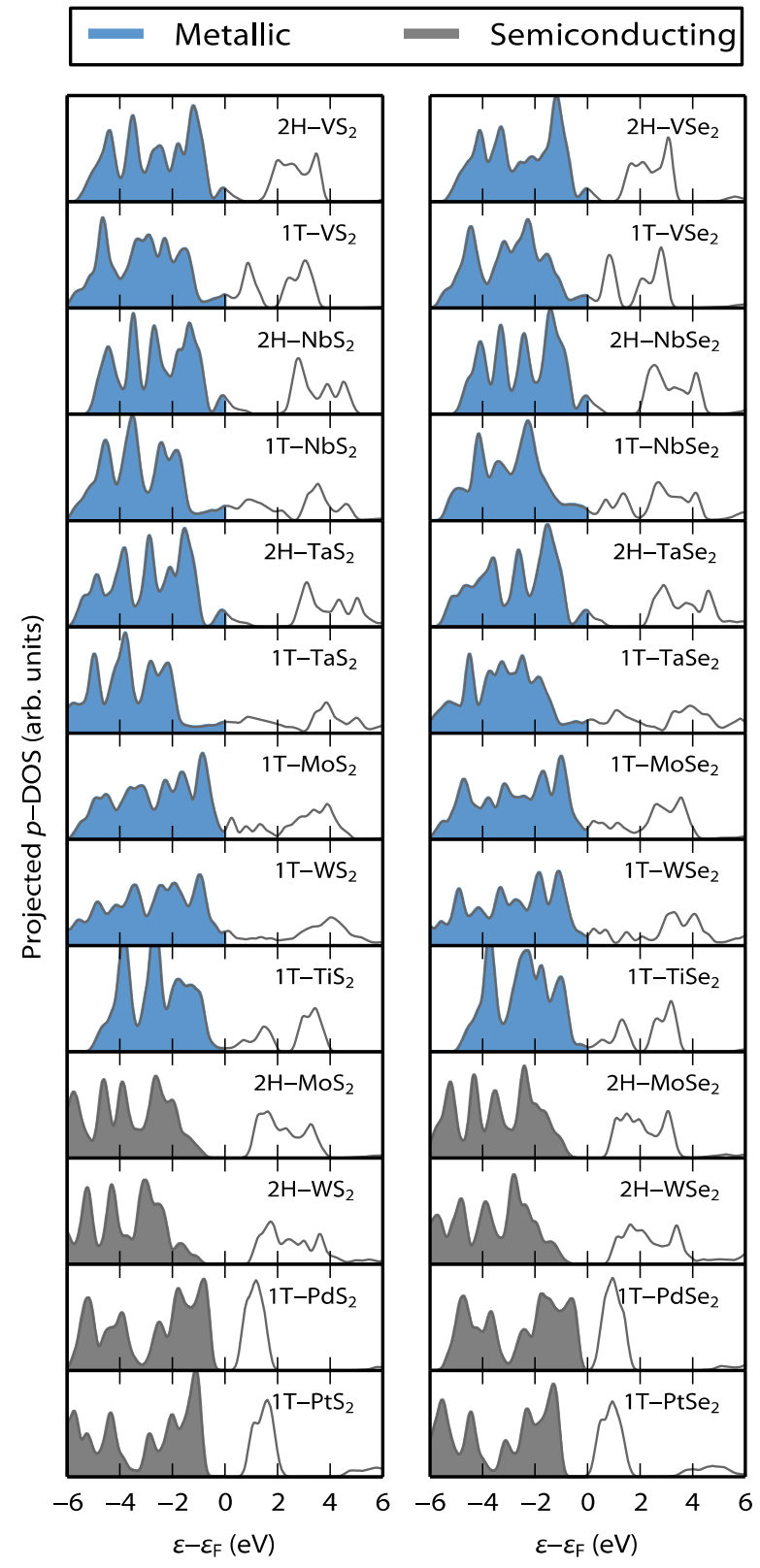

Figure 2. p-projected density of states on the chalcogen atom ( $\mathrm{S}$ or $\mathrm{Se}$ ) for TMD's relative to the Fermi level, $\varepsilon_{\mathrm{F}}$. The blue densities of states indicate that the basal plane is metallic whereas the grey ones are semiconducting. The basal planes of group 5 TMD's are metallic regardless of structure, whereas for group 6 TMD's (Mo and W), there is a change from semiconducting to metallic when the structure is changed from $2 \mathrm{H}$ to 1T. Group 4 TMD's are metallic in the 1T structure whereas group 10 TMD's are semiconducting in the $1 \mathrm{~T}$ structure. The shaded region indicates filling of the p-states up to the Fermi level, $\varepsilon_{\mathrm{F}}$.

In general, the strength of $\mathrm{HX}$ binding $\left(\Delta G_{\mathrm{HX}}, \mathrm{X}=\mathrm{S}\right.$ or Se) is inversely proportional to the $\mathrm{H}$ binding $\left(\Delta G_{\mathrm{H}}\right)$ onto the chalcogen X. This confirms the general understanding that a more unstable catalyst $\left(\Delta G_{\mathrm{HX}}\right.$ is more positive and $\mathrm{HX}$ binding is weaker) is also more reactive ( $\Delta G_{\mathrm{H}}$ is more negative and $\mathrm{H}$ binding is stronger). We have previously shown that the inverse linear scaling between $\Delta G_{\mathrm{H}}$ and $\Delta G_{\mathrm{X}}$ on TMS edges arises from the differences in the slopes of scaling between $\Delta G_{\mathrm{HX}}$ and $\Delta G_{\mathrm{H}}$ and the $d$-band center[32]. $\Delta G_{\mathrm{H}}$ vs. $\Delta G_{\mathrm{HX}}$ shows the same negative relationship because $\Delta G_{\mathrm{HX}}$ and $\Delta G_{\mathrm{X}}$ have a positive linear scaling[35,36]. Since hydrogen binding on the $\mathrm{X}$ atom is the first step in vacancy formation via desorption of $\mathrm{X}$ as $\mathrm{H}_{2} \mathrm{X}$, there is a relationship between how stable the surfaces are and how active they are. Fig. 3a shows this inverse relationship for the basal planes of the semiconducting TM sulfides and selenides, and Figure $3 \mathrm{~b}$ for the metallic TM sulfides and selenides. The differences in slopes amongst these relations arise from the presence of states near the Fermi level for the metallic TMD's compared to the semiconducting, as well as the more extended states of the selenium atoms compared to sulfur. These inverse relationships also indicate the role of the transition metal in determining the activity: the metal varies the strength of chalcogen binding onto the basal plane, which modifies the binding strength of $\mathrm{H}$ onto the chalcogen. Stronger HX binding leads to weaker $\mathrm{H}$ binding and weaker $\mathrm{HX}$ binding leads to stronger $\mathrm{H}$ binding. All $\Delta G_{\mathrm{H}}$ values on the surface 
are $>0 \mathrm{eV}$, so strategies for strengthening $\mathrm{H}$ binding to improve activity must come at the cost of making the catalyst less stable.

For the semiconducting surfaces, both the TM sulfides and selenides show weak hydrogen binding ( $\Delta G_{\mathrm{H}}$ ranges from $0.5 \mathrm{eV}$ to $2.5 \mathrm{eV}$ ) and a similar inverse relation between $\Delta G_{\mathrm{H}}$ and $\Delta G_{\mathrm{HX}}$. The optimal hydrogen adsorption free energy, $\Delta G_{\mathrm{H}}$ $=0 \mathrm{eV}$ is indicated by dashed lines. $\Delta G_{\mathrm{HX}}=0 \mathrm{eV}$, where desorption of $\mathrm{HX}$ on the basal plane as $\mathrm{H}_{2} \mathrm{X}$ becomes possible, is also indicated; this is where the basal plane begins to dissolve in solution. The data points approach $\Delta G_{\mathrm{H}}=0 \mathrm{eV}$ with increasing $\Delta G_{\mathrm{HX}}$, which indicates that $\mathrm{H}$ binding could be optimally tuned by weakening the HX binding (e.g. through transition metal doping). However, $\Delta G_{\mathrm{HX}}$ becomes positive far before any of the data points have a thermo-neutral $\Delta G_{\mathrm{H}}$, which indicates that even the surfaces that are least stable are not active for HER.
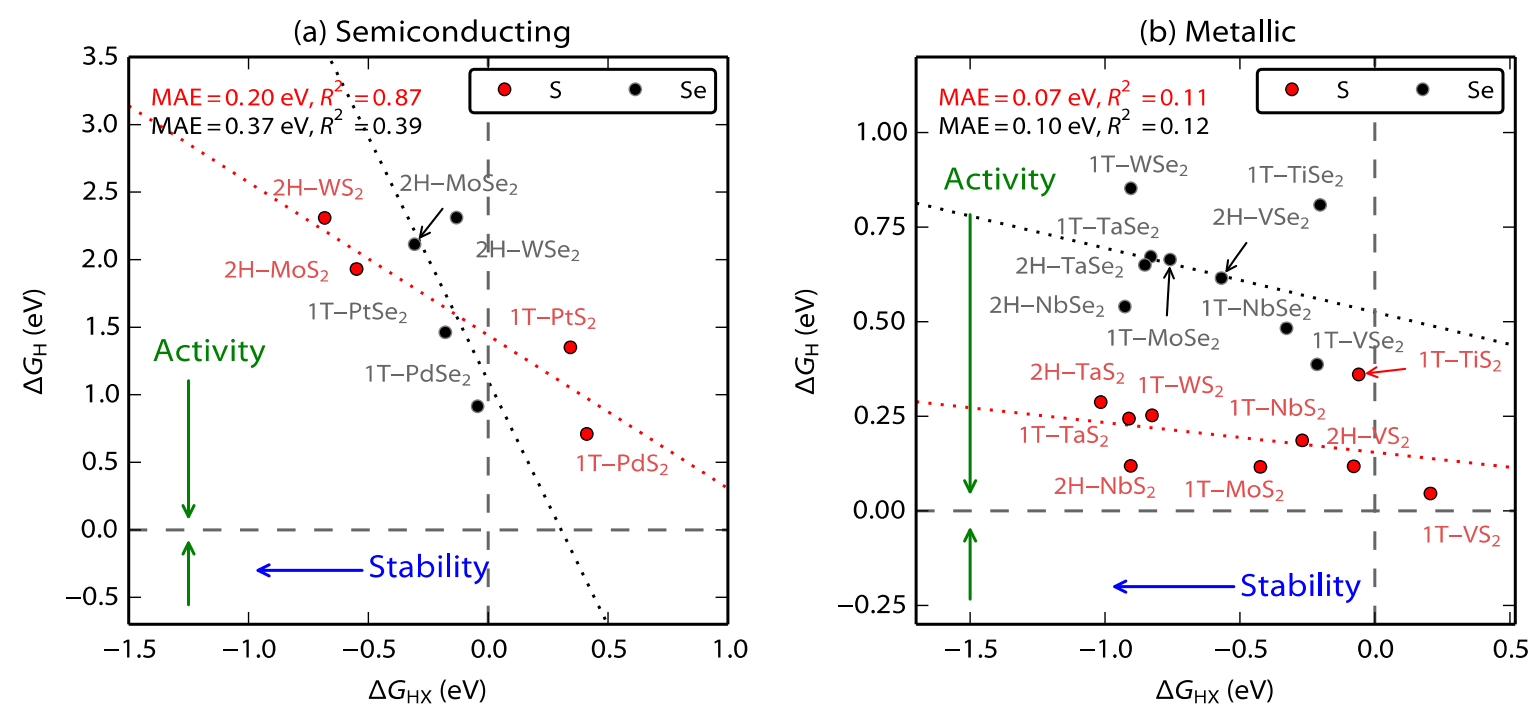

Figure 3. (a) Semiconducting single layered TMD basal planes. Plot of the hydrogen adsorption free energy, $\Delta G_{\mathrm{H}}$, as a function of the $\mathrm{HX}$ adsorption free energy, $\Delta G_{\mathrm{HX}}\left(\mathrm{X}=\mathrm{S}\right.$ or Se). All $\Delta G_{\mathrm{H}}$ are greater than $0.3 \mathrm{eV}$, indicating weak chemisorption. More reactive transition metals in TMD lead to stronger $\mathrm{X}$ binding and weaker $\mathrm{H}$ binding. The mean absolute error is large but the data spans a large range of $\Delta G_{\mathrm{H}}$. (b) Metallic single layered TMD basal planes. Plot of the $\Delta G_{\mathrm{H}} \mathrm{vs} \Delta G_{\mathrm{HX}}(\mathrm{X}=\mathrm{S}$ or Se). The metallic TM sulfides have $\Delta G_{\mathrm{H}}$ between $0.04 \mathrm{eV}$ and $0.3 \mathrm{eV}$, whereas the metallic TM selenides have $\Delta G_{\mathrm{H}}$ between $0.4 \mathrm{eV}$ and $1.0 \mathrm{eV}$. The dashed lines indicate $\Delta G_{\mathrm{H}}=0 \mathrm{eV}$, where hydrogen binding is optimal, and $\Delta G_{\mathrm{HX}}=0 \mathrm{eV}$, where $\mathrm{H}_{2} \mathrm{X}$ desorption becomes exergonic. The dotted lines are guides for the eye. The data spans a narrow range of $\Delta G_{\mathrm{H}}$ for both TMS and TMSe, so the $R^{2}$ value is low due to the scatter even though the mean absolute error is small.

The metallic TMDs (Figure $3 b)$ span a much narrower range of hydrogen adsorption free energies $(0.05 \mathrm{eV}$ to $1.0 \mathrm{eV})$ and show clearer distinctions between the TM sulfides and selenides. The inverse relation between HX binding and $\mathrm{H}$ binding also has a much weaker dependence. As seen previously for the metallic edges in $\mathrm{MoSe}_{2}$ and WSe ${ }_{2}$, the hydrogen binding on Se is generally weaker compared to that on S.[6] The metallic TM sulfides have hydrogen adsorption free energies closest to thermo-neutral (between $0.04 \mathrm{eV}$ and $0.3 \mathrm{eV}$ ), which indicates that they should all exhibit activity for HER. Beyond 1T$\mathrm{MoS}_{2}$ and $1 \mathrm{~T}-\mathrm{WS}_{2}$, which have already been shown to be active HER catalysts, $[2,14,21]$ the $1 \mathrm{~T}$ and $2 \mathrm{H}$ structures of group 5 TMD's are new possibilities for active HER catalysts. The metallic TM selenides have improved hydrogen binding over the semiconducting TM selenides, but are still considerably weaker than the metallic TM sulfides $\left(\Delta G_{\mathrm{H}}\right.$ are $\left.>0.3 \mathrm{eV}\right)$.

In terms of stability, the apparent lesser dependence of $\Delta G_{\mathrm{H}}$ on $\Delta G_{\mathrm{HX}}$, indicates that the role of the transition metal is much weaker in the metallic TMD's compared to the semiconducting TMD's. Even when HX binding is varied, changes in hydrogen binding are only over a narrow range. This suggests that improvements in activity via strengthening the $\mathrm{H}$ binding would come at a significant cost in the stability of the surface. This is especially problematic for metallic TM selenides, which already have hydrogen binding that is too weak. However, this also means that the stabilization of an already active catalyst could be a potential route towards developing an optimal catalyst (e.g. through transition metal doping).

The role of the structure appears significant insofar as it affects the metallic or semiconducting nature of the TMD catalyst; the inverse relationship does not strictly depend on the type of geometry. For the group 5 TMD's, which have metallic 1T and $2 \mathrm{H}$ structures, the change from $1 \mathrm{~T}$ to $2 \mathrm{H}$ represents a change in $\Delta G_{\mathrm{H}}$ of less than $0.08 \mathrm{eV}$. For Mo and W based TMD's, where there is a semiconductor-to-metallic transition from $2 \mathrm{H}$ to $1 \mathrm{~T}$, the change in $\Delta G_{\mathrm{H}}$ is approximately $-1.2 \mathrm{eV}$ for $\mathrm{TM}$ selenides and $-2.0 \mathrm{eV}$ for TM sulfides.

Scatter in the data results primarily from surface rearrangements due to the hydrogen adsorption, which was previously observed and studied for hydrogen adsorption on the edges of various transition metal dichalcogenides. [6,31] The small perturbations in the geometry of the chalcogen atoms contribute to both the stability of $\mathrm{H}$ in $\Delta G_{\mathrm{H}}$ as well as the stability of $\mathrm{HX}$ in $\Delta G_{\mathrm{HX}}$. The extended states of the Se also contribute to the significant restructuring for the TM selenides basal planes, 
which explains why the TM selenide data points have greater amounts of spread. The metallic TMD's appear to exhibit more scatter, which is due to the stronger interaction between $\mathrm{H}$ and $\mathrm{X}$ upon adsorption. However, since the range of $\Delta G_{\mathrm{H}}$ is so narrow, the mean absolute error is already less than $0.1 \mathrm{eV}$, which is well within the error of adsorption energies predicted using the BEEF-vdW functional[37]. For example, the metallic TMS basal planes have BEEF-vdW errors of $0.19 \mathrm{eV}$ for $\Delta G_{\mathrm{H}}$ and $0.23 \mathrm{eV}$ for $\Delta G_{\mathrm{HS}}$. However, since the scatter is still significant for the data, the lines act only as a rough guide for illustrating the general negative correlation.

Although the dependence of activity on stability was entirely determined by the metallicity and chalcogen, the Mo and W based TMD's exhibit a semiconductor to metallic transition when changing from the $2 \mathrm{H}$ to $1 \mathrm{~T}$ structure along with a large increase in hydrogen binding strength. This dramatic change in $\mathrm{H}$ binding arises from the clustering of the metal atoms into chains in the plane (shown as a repeating unit cell in blue in Figure 4). This behavior has been studied in detail previously for $1 \mathrm{~T}-\mathrm{ReS}_{2}$ and recently for Mo and W dichalcogenides. [19,38]It has been experimentally inferred[39] and then experimentally observed $[14,21,40]$ as a "zigzag" pattern for $1 \mathrm{~T}-\mathrm{WS}_{2}$ and $1 \mathrm{~T}-\mathrm{MoS}_{2}$, which results in an insulator to metal transition. The clustering results in two unique adsorption sites (circled in blue and black in Figure 4). The first site is a dangling chalcogen atom, similar to the edge sites of $2 \mathrm{H}$ structures, which have been well studied. [1,6,8,41-45] The second site is fully coordinated. The under-coordinated chalcogen atom is formed as the metal atoms in the plane move closer to form a metal-metal bond. For all group 5 TMD's of the 1T structure, hydrogen binding on the first site is stronger than the second site by approximately $0.7 \mathrm{eV}$. This is reflected in the p-projected density of states on each binding site (Figure 4), where there is a pronounced increase in the resonant states near the Fermi level on the first binding site.
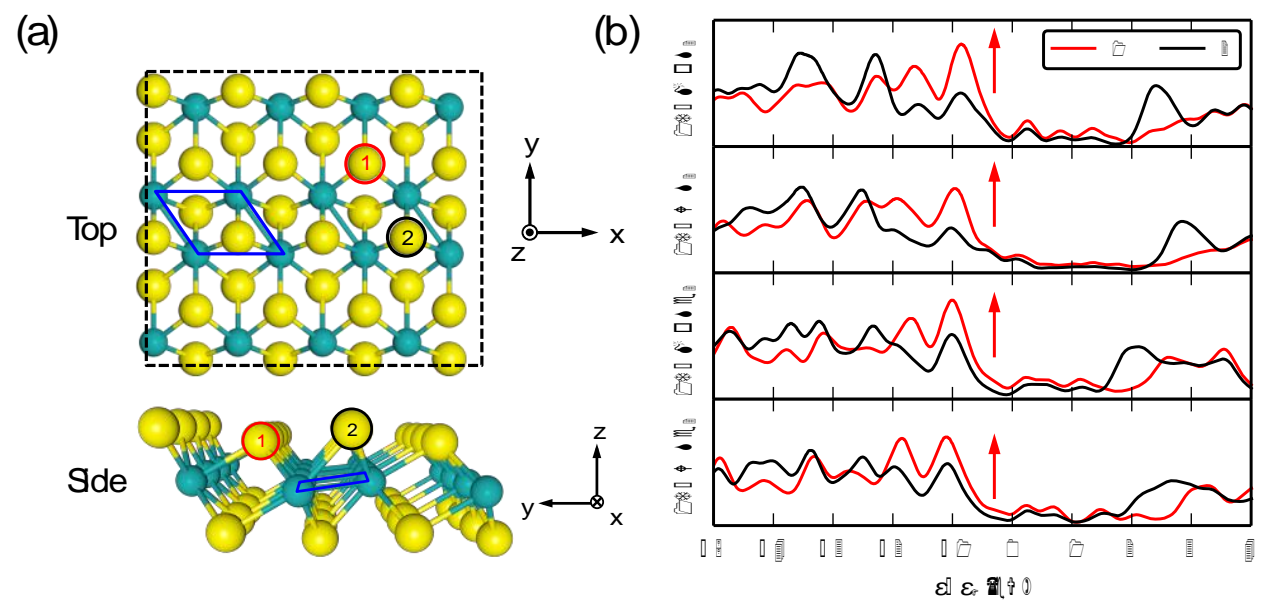

Figure 4. (a) Structural distortion present in $1 \mathrm{~T}-\mathrm{MoS}_{2}, 1 \mathrm{~T}-\mathrm{WS}_{2}, 1 \mathrm{~T}-\mathrm{MoSe}_{2}$, and 1T-WSe 2 . The "zigzag" structure caused by the metal-metal bond formation is highlighted by the blue unit cell, which repeats in the $x$ direction. The clustering results in two unique binding sites, with the first dangling chalcogen circled in red, and the second fully coordinated chalcogen circled in black. (b) The p-projected density of states on the chalcogen atom binding sites, showing the increased resonant states for the first binding site. The arrows indicate the increase in bonding states near the Fermi level in the decrease in anti-bonding states.

\subsection{Edge Sites}

We have thus far focused on the basal planes of the TMD catalysts, but the edge sites could play the dominant role in determining the overall activity, especially for the catalysts with inert basal planes. We again find that an approximate inverse relation defines the activity-stability behavior for all the edges, regardless of the composition, bulk metallicity, or structure (1T-edge, $2 \mathrm{H}$ M-edge, or $2 \mathrm{H} \mathrm{X}$-edge). The data points cross $\Delta G_{\mathrm{H}}=0 \mathrm{eV}$ before crossing the $\Delta G_{\mathrm{HX}}=0 \mathrm{eV}$ line, which indicates that an optimal hydrogen binding can be achieved for edges that are also stable against dissolution. This is in contrast to the basal planes, where $\Delta G_{\mathrm{HX}}>0 \mathrm{eV}$ and it becomes thermodynamically favorable for $\mathrm{HX}$ to desorb as $\mathrm{H}_{2} \mathrm{X}$ before hydrogen binding becomes optimal. Therefore, generating a high concentration of edge sites rather than through creating a reactive basal plane can make catalysts of optimal activity and stability. There is significant scatter in the data, which is due to a combination of factors: (1) the edges have varying steady-state X and H coverages; (2) there is a variable amount of reorganization on the edges due to hydrogen adsorption depending on the transition metal, where generally the X-edge and the 1Tedges show the most reorganization and hence the furthest deviations. The mobility of the chalcogen atoms on the edge also explains why the mean absolute errors (MAE) are significantly larger on the edges compared to the basal planes. We found previously with $\mathrm{MoS}_{2}$ based catalysts that using a consistent edge structure the scatter can be reduced significantly.[33]

We find that several catalysts that have active basal planes also have active edge sites. Notably, 1T-MoS $\mathrm{M}_{2}$ heen shown to have active basal planes as well as active edge sites in vertically aligned layers.[21,46] The activity and stability descriptors, $\Delta G_{\mathrm{H}}$ and $\Delta G_{\mathrm{HX}}$ respectively, for both the basal planes and all edges are summarized in Table 1. Generally, the edges are more reactive than the basal planes; however, for the catalysts with metallic basal planes, the binding energies can be similar. Regardless, there are more edges that are simultaneously active and stable, whereas the most reactive basal planes also start to become unstable. All $\Delta G$ values are summarized in Table 1. Catalyst supports have also been shown to modify 
adsorption on the basal planes and edges[31,47] and could play a role in modifying both the stability and hence activity of these catalysts. In reality, there may be systematic shifts in the $\Delta G_{\mathrm{H}}$ on either the basal plane or the edges, although the trends should remain consistent.

(a) TM Sulfide

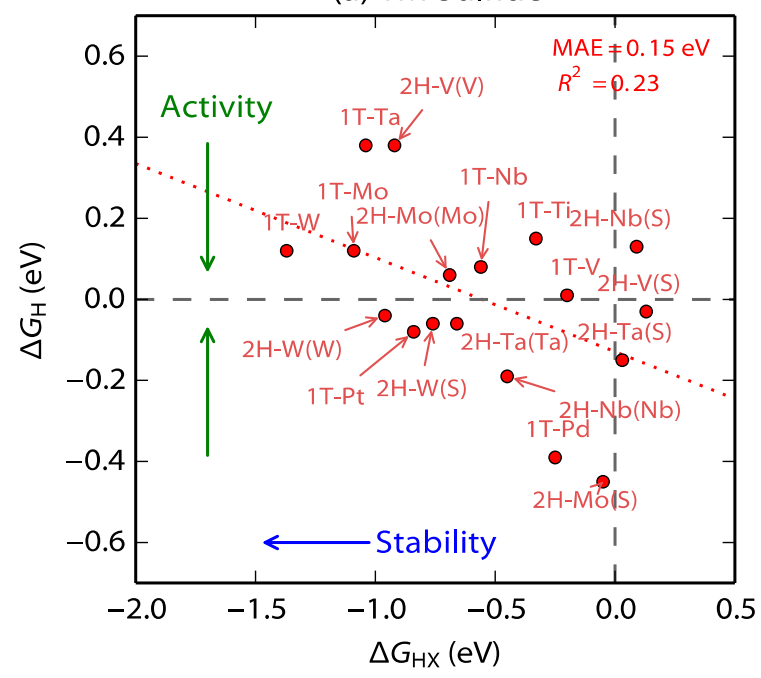

(a) TM Selenide

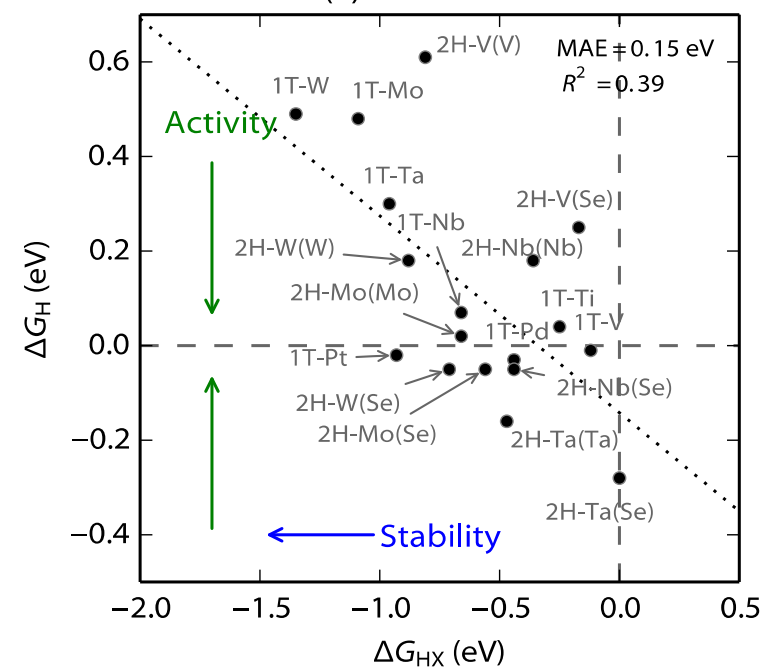

Figure 5. The differential hydrogen adsorption free energy $\Delta G_{\mathrm{H}}$ as a function of the $\mathrm{HX}$ adsorption free energy at a corrosion resistance of $10^{-6}$ bar $\mathrm{H}_{2} \mathrm{X}(\mathrm{X}=\mathrm{S}$ or $\mathrm{Se})$. The dashed lines indicate where $\Delta G_{\mathrm{H}}=0 \mathrm{eV}$ and where $\Delta G_{\mathrm{HX}}=0 \mathrm{eV}$. The dotted lines are guides for the eye. The data span a narrow range of $\Delta G_{\mathrm{H}}$ and the mean absolute errors are small, but the $R^{2}$ values are low due to the significant scatter.

\subsection{Hydrogen Coverage Dependence of $\Delta G_{\mathrm{H}}$ on the Edge Sites}

In contrast to the basal planes, where hydrogen binding is always weak $\left(\Delta G_{\mathrm{H}}>0 \mathrm{eV}\right)$, several of the edges have $\Delta G_{\mathrm{H}}<0 \mathrm{eV}$ at the lowest hydrogen coverage and hence, the steady-state coverage of hydrogen needs to be explicitly considered in our study. Generally, the hydrogen binding weakens incrementally with increasing hydrogen coverage (Fig. 6). Unlike the pure transition metals, the weakening in hydrogen binding does not arise solely from the repulsion between interacting adsorbates. The large distances between the hydrogen atoms at low coverages [4] and the rearrangement of the sulfur atoms when hydrogen is adsorbed [6,31] suggest instead that the weakening of $\mathrm{H}$ binding upon successive $\mathrm{H}$ adsorption arises from the limited number of metallic states and corresponding changes in geometry. This incremental weakening of hydrogen binding occurs regardless of the degree of basal plane metallicity. This is the reason why a large amount of the edge sites have steady-state hydrogen adsorption free energies that are very close to $0 \mathrm{eV}$.

The variation in hydrogen binding due to coverage raises the concern of whether or not our approach of using a single hydrogen binding energy at the steady-state coverage is adequate for describing the trends in activity. We discuss this issue in the context of the possible HER mechanism in the next section. 


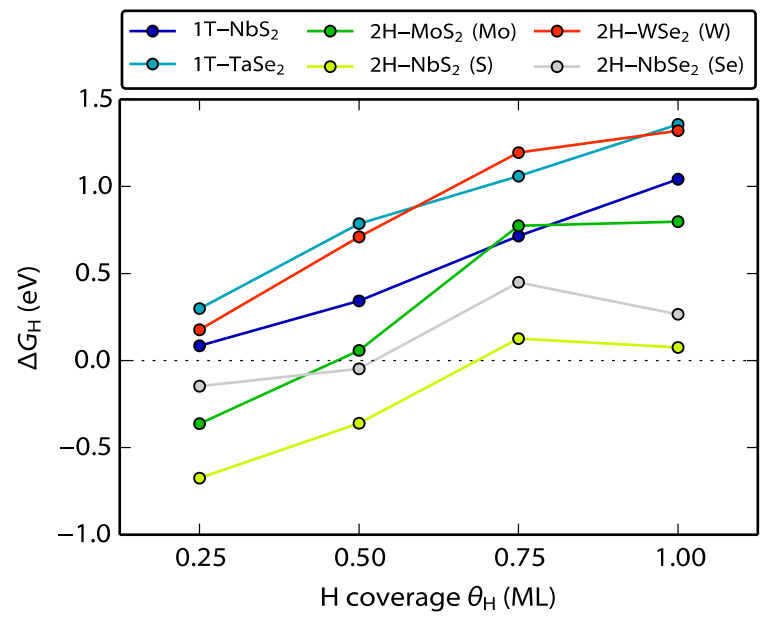

Figure 6. The change in hydrogen adsorption free energy $\Delta G_{\mathrm{H}}$ as a result of coverage for several representative TMD edges. One example is shown from each structure $(1 \mathrm{~T}$ and $2 \mathrm{H})$, each chalcogen ( $\mathrm{S}$ and $\mathrm{Se})$, and each type of edge (1T, $2 \mathrm{H}-\mathrm{M}$, and $2 \mathrm{H}-\mathrm{X})$. The parentheses indicate the type of edge surface on the $2 \mathrm{H}$ structures.

Table 1. Summary of the $\Delta G_{\mathrm{H}}$ and $\Delta G_{\mathrm{HX}}$ values for TMD catalysts. Data for the $2 \mathrm{H}-\mathrm{MoS}_{2}, 2 \mathrm{H}-\mathrm{WS}_{2}, 2 \mathrm{H}-\mathrm{MoSe}{ }_{2}$, and $2 \mathrm{H}-$ $\mathrm{WSe}_{2}$ were taken from previous studies. $[6,31]$

\begin{tabular}{|c|c|c|c|c|c|c|c|c|c|c|}
\hline \multirow{3}{*}{ Compound } & \multicolumn{6}{|c|}{$2 \mathrm{H}$} & \multicolumn{4}{|c|}{$1 \mathrm{~T}$} \\
\hline & \multicolumn{2}{|c|}{ Basal } & \multicolumn{2}{|c|}{ M-edge } & \multicolumn{2}{|c|}{ X-edge } & \multicolumn{2}{|c|}{ Basal } & \multicolumn{2}{|c|}{ Edge } \\
\hline & $\Delta G_{\mathrm{HX}}$ & $\Delta G_{\mathrm{H}}$ & $\Delta G_{\mathrm{HX}}$ & $\Delta G_{\mathrm{H}}$ & $\Delta G_{\mathrm{HX}}$ & $\Delta G_{\mathrm{H}}$ & $\Delta G_{\mathrm{HX}}$ & $\Delta G_{\mathrm{H}}$ & $\Delta G_{\mathrm{HX}}$ & $\Delta G_{\mathrm{H}}$ \\
\hline $\mathrm{TiS}_{2}$ & - & - & - & - & - & - & -0.09 & 0.38 & -0.33 & 0.15 \\
\hline $\mathrm{VS}_{2}$ & -0.08 & 0.12 & -0.92 & 0.38 & -0.20 & 0.01 & 0.21 & 0.05 & -0.20 & 0.01 \\
\hline $\mathrm{NbS}_{2}$ & -0.90 & 0.12 & -0.45 & -0.19 & 0.09 & 0.13 & -0.27 & 0.19 & -0.56 & 0.08 \\
\hline $\mathrm{TaS}_{2}$ & -1.02 & 0.29 & -0.66 & -0.06 & 0.03 & -0.15 & -0.91 & 0.24 & -1.04 & 0.38 \\
\hline $\mathrm{MoS}_{2}$ & -0.55 & 1.92 & -0.69 & 0.06 & -0.05 & -0.45 & -0.42 & 0.12 & -1.09 & 0.12 \\
\hline $\mathrm{WS}_{2}$ & -0.68 & 2.23 & -0.96 & -0.04 & -0.76 & -0.06 & -0.83 & 0.25 & -1.37 & 0.12 \\
\hline $\mathrm{PdS}_{2}$ & - & - & - & - & - & - & 0.41 & 0.71 & -0.25 & -0.39 \\
\hline $\mathrm{PtS}_{2}$ & - & - & - & - & - & - & 0.34 & 1.35 & -0.84 & -0.08 \\
\hline $\mathrm{TiSe}_{2}$ & - & - & - & - & - & - & -0.20 & 0.81 & -0.25 & 0.04 \\
\hline $\mathrm{VSe}_{2}$ & -0.57 & 0.62 & -0.81 & 0.61 & -0.17 & 0.25 & -0.21 & 0.39 & -0.17 & 0.25 \\
\hline $\mathrm{NbSe}_{2}$ & -0.93 & 0.54 & -0.36 & 0.18 & -0.44 & -0.28 & -0.33 & 0.48 & -0.66 & 0.07 \\
\hline $\mathrm{TaSe}_{2}$ & -0.85 & 0.65 & -0.47 & -0.16 & 0.00 & -0.10 & -0.83 & 0.67 & -0.96 & 0.30 \\
\hline $\mathrm{MoSe}_{2}$ & -0.31 & 2.13 & -0.66 & 0.02 & -0.56 & -0.05 & -0.76 & 0.66 & -1.09 & 0.48 \\
\hline $\mathrm{WSe}_{2}$ & -0.13 & 2.31 & -0.88 & 0.17 & -0.71 & -0.05 & -0.90 & 0.85 & -1.35 & 0.49 \\
\hline $\mathrm{PdSe}_{2}$ & - & - & - & - & - & - & -0.04 & 0.89 & -0.44 & -0.03 \\
\hline $\mathrm{PtSe}_{2}$ & - & - & - & - & - & - & -0.18 & 1.44 & -0.93 & -0.02 \\
\hline
\end{tabular}

\section{POSSIBLE MECHANISM FOR HYDROGEN EVOLUTION}

The hydrogen evolution reaction could proceed through either the Volmer-Tafel or the Volmer-Heyrovsky mechanism $[9,24,25]$, and it has not yet been determined which mechanism is the relevant one for TMD catalysts. In order to determine the activation barriers for the Volmer and Heyrovsky steps, an explicit consideration of the interfacial solvent is required; however, since the Tafel mechanism involves only an associative desorption from the surface, it could be readily modeled by ignoring the interfacial water molecules as a first approximation. We have used the Nudged Elastic Band (NEB) method[34] to determine the activation barriers for the Tafel reaction on the $1 \mathrm{~T}-\mathrm{MoS}_{2}$ basal plane and the $2 \mathrm{H}-\mathrm{MoS}_{2} \mathrm{Mo}$-edge, since these 
surfaces have been shown experimentally to have high activity for HER.[8,21] For both the basal plane of $1 \mathrm{~T}-\mathrm{MoS}_{2}$ and the Mo-edge of $2 \mathrm{H}-\mathrm{MoS}_{2}$ (Fig. 7 and Fig. 8), the activation barrier is $\sim 1.5 \mathrm{eV}$ for $\mathrm{H}_{2}$ desorption and is thus insurmountable under room temperature, electrochemical conditions. Since it has been shown from experiments that $1 \mathrm{~T}-\mathrm{MoS}_{2}$ and $2 \mathrm{H}-\mathrm{MoS}_{2}$ require only a small overpotential, it is thus highly unlikely that HER on these materials proceeds via the Volmer-Tafel route. Although we determined the barriers in the absence of a solvation layer, the minimal interaction between hydrogen and water suggests that the Tafel route would not be any more accessible with solvation. From the NEB calculations, the large barrier is seen to arise from the $\sim 3.3 \AA$ distance between neighboring $\mathrm{S}$ atoms. At the transition state, the $\mathrm{S}-\mathrm{S}$ distance is reduced to $\sim$ $3.0 \AA$ in order to allow the $\mathrm{H}-\mathrm{H}$ distance to be small enough for a bond to form. This represents a significant distortion of the surface geometry. Since similar distances for all the surfaces on all the TMD's separate the chalcogen atoms, we conclude that the Volmer-Heyrovsky mechanism is the more likely mechanism across all surfaces for all the TMD's. The likelihood of the Heyrovsky mechanism also provides validation for our method of using $\Delta G_{\mathrm{H}}$ values to approximate HER activity. Since only one adsorption site is involved in this mechanism, only one $\mathrm{H}$ adsorption energy corresponding to the steady state coverage is required to describe activity. Explicit consideration of solvation at the surface is necessary in order to accurately model the Volmer-Heyrovsky route and further confirm whether or not it is the relevant route for HER. This will be considered in future studies.

(a)

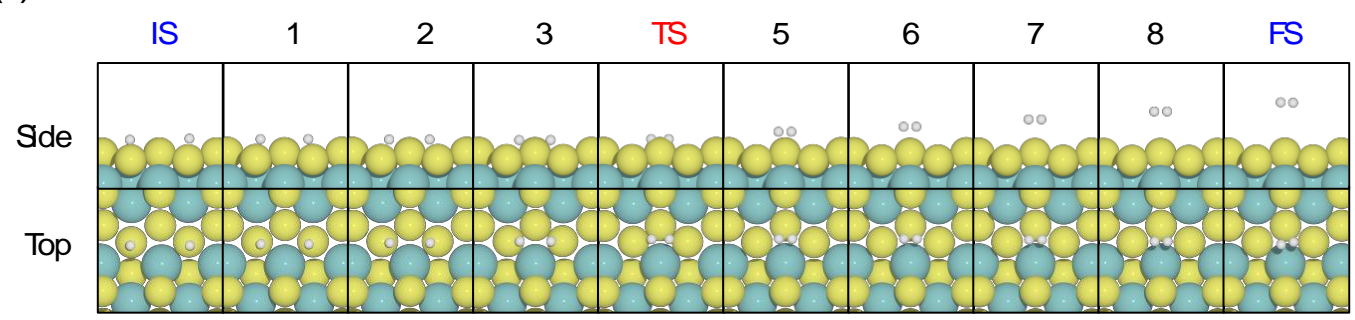

(b)

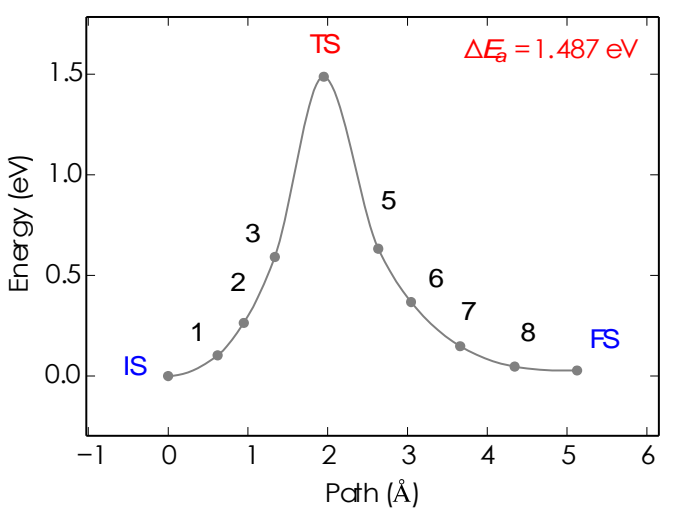

(c)

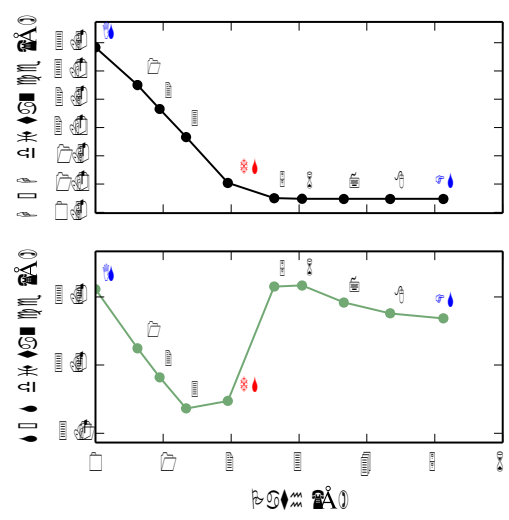

Figure 7. Nudged Elastic Band (NEB) calculation results for $\mathrm{H}_{2}$ desorption via the Tafel mechanism on the $1 \mathrm{~T}-\mathrm{MoS} 2$ basal plane. (a) Structures corresponding to each point on the reaction pathway in the NEB calculations. The images are ordered from the initial state (IS), to the transition state (TS), then to the final state (FS); (b) the activation barrier as determined by NEB; (c) the change in $\mathrm{H}-\mathrm{H}$ bond distance throughout the reaction pathway. The labels correspond to the image number. 
(a)



(b)

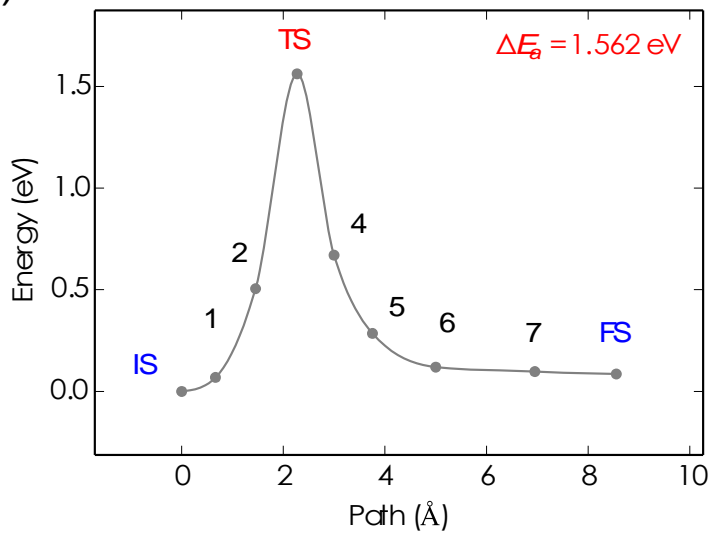

(c)

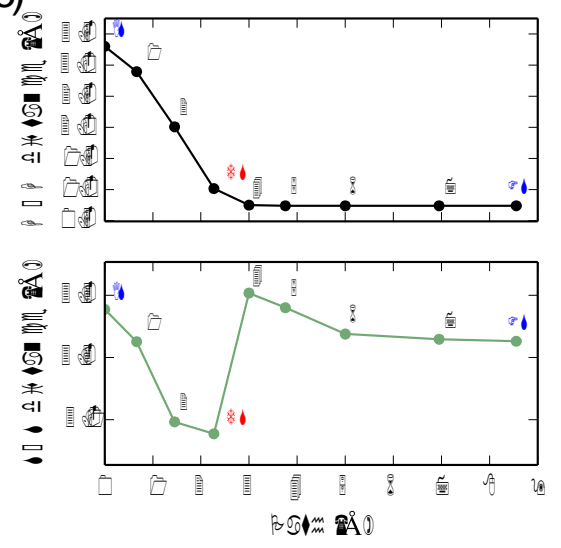

Figure 8. Nudged Elastic Band (NEB) calculation results for $\mathrm{H}_{2}$ desorption via the Tafel mechanism on the Mo-edge of 2H$\mathrm{MoS}_{2}$. The images are ordered from the initial state (IS), to the transition state (TS), then to the final state (FS); (a) Structures corresponding to each point on the reaction pathway in the NEB calculations; (b) the activation barrier as determined by NEB; (c) the change in $\mathrm{H}-\mathrm{H}$ bond distance throughout the reaction pathway.

\section{CONCLUSIONS}

In summary, we have computationally screened the basal planes and edges of several TMD's for their activity and stability and we established the role of the transition metal, chalcogen, and structure in modifying the activity and stability. Within the class of catalysts that have basal planes that are either semiconducting or metallic, the transition metal serves as a modifier of how strongly the chalcogen is bound, which inversely relates to how strongly the hydrogen is bound at the surface. Hydrogen binding onto selenides is weaker than onto sulfides, although the effect is much less pronounced for the semiconducting TMD's. For the basal plane, the structure is significant insofar as it changes the semiconducting or metallic nature of the TMD, with metallic catalysts having much stronger hydrogen binding. The metallic TM sulfide basal planes all show hydrogen adsorption free energies close to thermo-neutral, which suggests they could be active HER catalysts. The edges were all found to be metallic regardless of the metallicity of the basal plane, and an inverse relationship approximately determines their activity and stability. Despite the possibility of reactive basal planes in TMD catalysts, their activities are severely limited by their stabilities. For the TMD catalysts considered, optimal hydrogen binding is not achievable for stable basal planes whereas it is possible for the edges. Through the observed inverse relationships between $\Delta G_{\mathrm{H}}$ and $\Delta G_{\mathrm{HX}}$, we also establish the limitations on the hydrogen evolution activity on TMD's in general: weakly bound chalcogen atoms on the basal plane lead to improved activity, but at a significant cost in stability. We have also determined that the Tafel mechanism has an insurmountably high barrier on both the $2 \mathrm{H}-\mathrm{MoS}_{2}$ Mo-edge and the $1 \mathrm{~T}-\mathrm{MoS}_{2}$ basal plane, suggesting that the TMD catalysts proceed through the Volmer-Heyrovsky route. Further work involving an explicit consideration of the solvation layer will be required to confirm whether or not this is the case.

\section{ASSOCIATED CONTENT}

Supporting Information. Computational details, calculated lattice parameters, and a summary of the stable surfaces.

\section{AUTHOR INFORMATION}

\section{Corresponding Author}

*Frank Abild-Pedersen 
Tel: +1 650-926-2480

E-mail: abild@slac.stanford.edu

\section{Author Contributions}

The manuscript was written through contributions of all authors. All authors have given approval to the final version of the manuscript.

\section{ACKNOWLEDGMENT}

We acknowledge support from the Center on Nanostructuring for Efficient Energy Conversion (CNEEC) at Stanford University, an Energy Frontier Research Center funded by the U.S. Department of Energy, Office of Basic Energy Sciences under award number DE-SC0001060. F.A.-P. and J.K.N acknowledge financial support from the U.S. Department of Energy (DOE), Office of Basic Energy Sciences to the SUNCAT Center for Interface Science and Catalysis. C.T. acknowledges support from the National Science Foundation Graduate Research Fellowship Program (GRFP) Grant DGE-114747. K.C. acknowledges support from the Air Force Office of Scientific Research through the MURI program under AFOSR Award No. FA9550-10-1-0572.

\section{REFERENCES}

[1] M.V. Bollinger, K.W. Jacobsen, J.K. Nørskov, Phys. Rev. B 67 (2003) 085410.

[2] M.S. Dresselhaus, I.L. Thomas, Nature 414 (2001) 332.

[3] J.A. Wilson, A.D. Yoffe, Adv. Phys. 18 (1969) 193.

[4] B. Hinnemann, P.G. Moses, J.L. Bonde, K.P. Jørgensen, J.H. Nielsen, S. Horch, I. Chorkendorff, J.K. Nørskov, J. Am. Chem. Soc. 127 (2005) 5308.

[5] M. Chhowalla, H.S. Shin, G. Eda, L.-J. Li, K.P. Loh, H. Zhang, Nat. Chem. 5 (2013) 263.

[6] C. Tsai, K. Chan, F. Abild-Pedersen, J.K. Nørskov, Phys. Chem. Chem. Phys. 16 (2014) 13156.

[7] J.L. Bonde, P.G. Moses, T.F. Jaramillo, J.K. Nørskov, I. Chorkendorff, Faraday Discuss. 140 (2008) 219.

[8] T.F. Jaramillo, K.P. Jørgensen, J.L. Bonde, J.H. Nielsen, S. Horch, I. Chorkendorff, Science 317 (2007) 100.

[9] J.K. Nørskov, T. Bligaard, Á. Logadóttir, J.R. Kitchin, J.G. Chen, S. Pandelov, U. Stimming, J. Electrochem. Soc. 152 (2005) $\mathrm{J} 23$.

[10] J. Kibsgaard, Z. Chen, B.N. Reinecke, T.F. Jaramillo, Nat. Mater. 11 (2012) 963.

[11] D. Kong, H. Wang, J.J. Cha, M. Pasta, K.J. Koski, J. Yao, Y. Cui, Nano Letters 13 (2013) 1341.

[12] H. Wang, H. Wang, D. Kong, D. Kong, P. Johanes, P. Johanes, J.J. Cha, G. Zheng, G. Zheng, K. Yan, N. Liu, Y. Cui, Nano Letters (2013).

[13] X. Lu, M.I.B. Utama, J. Lin, X. Gong, J. Zhang, Y. Zhao, S.T. Pantelides, J. Wang, Z. Dong, Z. Liu, W. Zhou, Q. Xiong, Nano Letters 14 (2014) 2419.

[14] D. Voiry, H. Yamaguchi, J. Li, R. Silva, D.C.B. Alves, T. Fujita, M. Chen, T. Asefa, V.B. Shenoy, G. Eda, M. Chhowalla, Nat. Mater. (2013).

[15] J. Yang, D. Voiry, S.J. Ahn, D. Kang, A.Y. Kim, M. Chhowalla, H.S. Shin, Angew. Chem. Int. Ed. 52 (2013) 13751.

[16] K. Chan, C. Tsai, H.A. Hansen, J.K. Nørskov, ChemCatChem 6 (2014) 1899.

[17] M. Asadi, B. Kumar, A. Behranginia, B.A. Rosen, A. Baskin, N. Repnin, D. Pisasale, P. Phillips, W. Zhu, R. Haasch, R.F. Klie, P. Král, J. Abiade, A. Salehi-Khojin, Nat Commun 5 (2014) 4470.

[18] R. Michalsky, Y.-J. Zhang, A.A. Peterson, ACS Catal. (2014) 1274.

[19] M. Kertesz, R. Hoffmann, J. Am. Chem. Soc. 106 (1984) 3453.

[20] H.I. Karunadasa, E. Montalvo, Y. Sun, M. Majda, J.R. Long, C.J. Chang, Science 335 (2012) 698.

[21] D. Voiry, M. Salehi, R. Silva, T. Fujita, M. Chen, Nano Letters 13 (2013) 6222.

M.A. Lukowski, A.S. Daniel, F. Meng, A. Forticaux, L. Li, S. Jin, J. Am. Chem. Soc. (2013).

M.A. Lukowski, A.S. Daniel, C.R. English, F. Meng, A. Forticaux, R.J. Hamers, S. Jin, Energy Environ. Sci. (2014).

S. Trasatti, J. Electroanal. Chem. 33 (1971) 351.

S. Trasatti, in:, H. Gerischer, C.W. Tobias (Eds.), Advances in Electrochemical Science and Engineering, Volume 2, Wiley, n.d. B. Hinnemann, J.K. Nørskov, H. Topsøe, J. Phys. Chem. B 109 (2005) 2245.

R. Parsons, Trans. Faraday Soc. 54 (1958) 1053.

J. Greeley, M. Mavrikakis, Nat. Mater. 3 (2004) 810.

M. Pourbaix, Atlas D'equilibres Electrochimiques, Gauthier-Villars, Paris, 1963.

H.A. Hansen, J. Rossmeisl, J.K. Nørskov, Phys. Chem. Chem. Phys. 10 (2008) 3722.

C. Tsai, F. Abild-Pedersen, J.K. Nørskov, Nano Letters 14 (2014) 1381.

C. Tsai, K. Chan, J.K. Nørskov, F. Abild-Pedersen, J. Phys. Chem. Lett. 5 (2014) 3884.

C. Tsai, K. Chan, J.K. Nørskov, F. Abild-Pedersen, Catal. Sci. Technol. 5 (2015) 246.

G. Henkelman, B.P. Uberuaga, H. Jónsson, J. Chem. Phys. 113 (2000) 9901.

[35] E.M. Fernández, P.G. Moses, A. Toftelund, H.A. Hansen, J.I. Martínez, F. Abild-Pedersen, J. Kleis, B. Hinnemann, J. Rossmeisl, T. Bligaard, J.K. Nørskov, Angew. Chem. Int. Ed. 47 (2008) 4683.

[36] E.M. Fernández, P.G. Moses, A. Toftelund, H.A. Hansen, J.I. Martínez, F. Abild-Pedersen, J. Kleis, B. Hinnemann, J. Rossmeisl, T. Bligaard, Angew. Chem. 120 (2008) 4761.

[37] J. Wellendorff, K.T. Lundgaard, A. Møgelhøj, V. Petzold, D.D. Landis, J.K. Nørskov, T. Bligaard, K.W. Jacobsen, Phys. Rev. B 85 (2012) 235149.

[38] K.-A.N. Duerloo, Y. Li, E.J. Reed, Nat Commun 5 (2014).

[39] J. Heising, M.G. Kanatzidis, J. Am. Chem. Soc. 121 (1999) 638. 
[40] L. Wang, Z. Xu, W. Wang, X. Bai, J. Am. Chem. Soc. 136 (2014) 6693.

[41] P. Raybaud, J. Hafner, G. Kresse, H. Toulhoat, Surf. Sci. 407 (1998) 237.

[42] H. Schweiger, P. Raybaud, G. Kresse, H. Toulhoat, Journal of Catalysis 207 (2002) 76.

[43] Á. Logadóttir, P.G. Moses, B. Hinnemann, N.-Y. Topsøe, K.G. Knudsen, H. Topsøe, J.K. Nørskov, Catal. Today 111 (2006) 44.

[44] M.V. Bollinger, J.V. Lauritsen, K.W. Jacobsen, J.K. Nørskov, S. Helveg, F. Besenbacher, Phys. Rev. Lett. 87 (2001).

[45] H.G. Füchtbauer, A.K. Tuxen, P.G. Moses, Phys. Chem. Chem. Phys. (2013).

[46] H. Wang, Z. Lu, D. Kong, J. Sun, T.M. Hymel, Y. Cui, ACS Nano (2014) 140414151348004.

[47] W. Chen, E.J.G. Santos, W. Zhu, E. Kaxiras, Z. Zhang, Nano Letters 13 (2013) 509. 